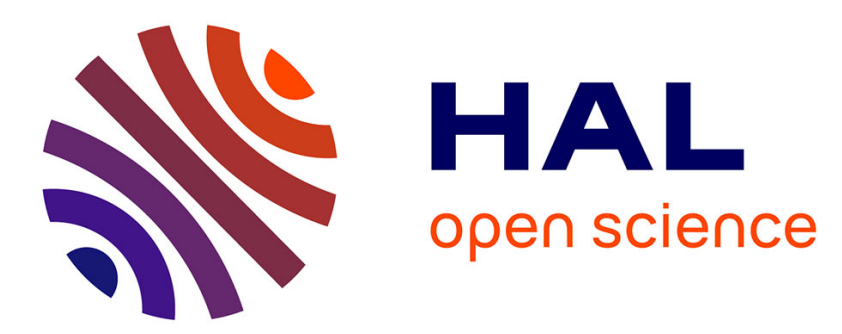

\title{
Towards Co-located TSCH Networks: An Inter-network Interference Perspective
}

Tim van Der Lee, Saleem Raza, George Exarchakos, Mesut Günes

\section{To cite this version:}

Tim van Der Lee, Saleem Raza, George Exarchakos, Mesut Günes. Towards Co-located TSCH Networks: An Inter-network Interference Perspective. GLOBECOM 2018 - 2018 IEEE Global Communications Conference, Dec 2018, Abu Dhabi, France. pp.1-6, 10.1109/GLOCOM.2018.8647867. hal-02915630

\section{HAL Id: hal-02915630 \\ https://hal.science/hal-02915630}

Submitted on 14 Aug 2020

HAL is a multi-disciplinary open access archive for the deposit and dissemination of scientific research documents, whether they are published or not. The documents may come from teaching and research institutions in France or abroad, or from public or private research centers.
L'archive ouverte pluridisciplinaire HAL, est destinée au dépôt et à la diffusion de documents scientifiques de niveau recherche, publiés ou non, émanant des établissements d'enseignement et de recherche français ou étrangers, des laboratoires publics ou privés. 


\title{
Towards Co-located TSCH Networks: An Inter-network Interference Perspective
}

\author{
Tim van der Lee*, Saleem Raza ${ }^{\dagger}$, George Exarchakos*, and Mesut Güneş ${ }^{\dagger}$ \\ *Eindhoven University of Technology \\ P.O. Box 513, 5600MB Eindhoven, the Netherlands \\ ${ }^{\dagger}$ Communication and Networked Systems (ComSys), Faculty of Computer Science, \\ Otto-von-Guericke University, Universitätsplatz 2, 39106 Magdeburg, Germany \\ emails: $\{t . l e e, g . e x a r c h a k o s\} @ t u e . n l,\{$ saleem.raza, mesut.guenes\}@ovgu.de
}

\begin{abstract}
The future Internet of Things (IoT) is expected to be dense with multiple independently administered colocated wireless sensor networks operating in close proximity, especially in e-health and industrial automotive applications. Recent advances in wireless technologies and standards for IoT communication such as IEEE 802.15.4, WLAN, ZigBEE, and WirelessHART make use of unlicensed bands and several networks may co-exist in close locations. This results co-located networks acting as interferer and interference victim for one another, hence affecting network reliability and energy costs. In this regard, the case of IEEE 802.15.4-time-slotted channel hopping (TSCH) protocol is of particular importance because of its widespread acceptance as a reliable MAC layer protocol for industrial low-power IoT.

This paper studies closely co-located TSCH networks from the interference perspective and shows how it impacts the network performance in terms of successful communication and periodic communication blockage. Our results demonstrate that co-located TSCH networks periodically interfere with one another if they do not cooperate. The impact of inter-network interference can be reduced when there are less time-sources. Exploiting 16 channels of the IEEE 802.15.4-TSCH with channel hopping mechanism greatly reduces inter-network interference.
\end{abstract}

\section{INTRODUCTION}

Recent advances in the Internet of Things (IoT) push the need to define new protocols to meet the requirements of its dense deployments. Real-world deployments of industrial IoT are expected to integrate multi-vendor solutions. Most of these solutions prefer wireless communication based on different standards such as IEEE 802.15.4 [1], WLAN, WirelessHART [2], and ISA100.11a [3]. This co-existence of network protocols is prone to creating interference, particularly in urban residential, smart city [4], and industrial scenarios [5]. Due to high heterogeneity of devices and their ownership, interference across co-located networks emerges as a barrier to guarantee reliability and low energy consumption. Interference indeed causes packet loss which increases the energy for retransmission, hence affects the communication reliability. One such interesting case is the time-slotted channel hopping (TSCH) [1] medium access control (MAC) protocol which has got widespread acceptance from both academia and industry as an ideal candidate protocol because

Note: The first two authors contributed equally to this work. of its very low energy consumption and high reliability claims [6], [7]. Nowadays, most of the evaluation of communication protocols is done in standalone networks. Very little attention is devoted to evaluating their behavior in co-located networks. Though, their performance may be good in an isolated network, yet they may present performance issues when different instances of these protocols are operating in co-located networks in the same physical space.

The focus of this paper is to study the impact of internetwork interference and behavior of interaction among closely co-located IEEE 802.15.4-TSCH networks. To achieve this, we investigate the impact of inter-network interference between two networks when introducing a clock drift between them. We analyze the impact of channel hopping on inter-network interference. We also study scalability perspectives and analyze the impact when the number of networks increases and when the size of the network grows. Our results demonstrate that, since TSCH networks are timesynchronized, co-locating networks interfere periodically and this period depends on the clock drift between the two networks. We show that when augmenting the number of nodes in the network or increasing the number of networks has more impact on inter-network interference than keeping the minimum number of networks possible. Also, the channel hopping, already known to mitigate external perturbations [8], greatly contributes to reducing inter-network interference.

The rest of the paper is organized as follows. We give an overview of the technical detail of the $\mathrm{TSCH}$ protocol and related work in Section II and Section III respectively. Section IV presents scenario and experiment set-up. Results are discussed in Section $\mathrm{V}$ and a conclusion is drawn in Section VI.

\section{BACKGROUND}

This section gives a brief technical overview of the TSCH communication protocol and its working mechanism.

\section{A. Overview of TSCH Communication Protocol}

Time slotted channel hopping (TSCH) is one of the MAC modes of the IEEE 802.15.4e-2015 [1] standard which is an amendment to the original IEEE 802.15.4 [9] standard. TSCH MAC mode has received increased attention for IoT, 


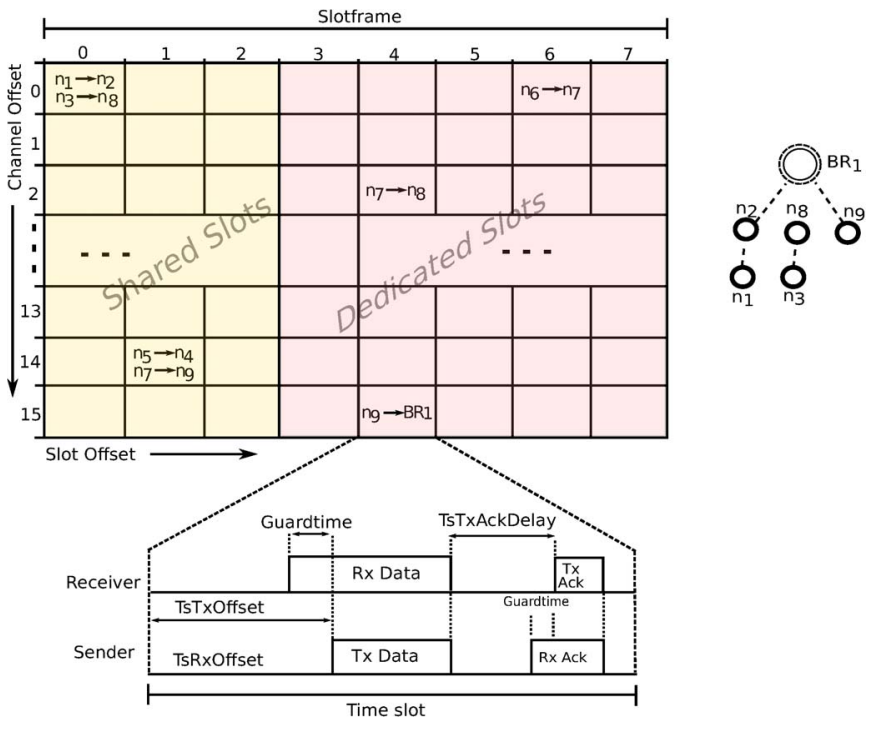

Figure 1. On the right, TSCH slotframe showing dedicated and shared slots for the associated topology on the left. It also shows typical sequence of events taking place within a slot when a sender sends a frame to the receiver.

especially in industrial deployments, due to its time-slotted structure and channel hopping which offer enhanced communication reliability and very low power consumption [10], [6], [7]. Operating with other constrained protocols such as 6LoWPAN [11] or IPv6 routing protocol for low-power and lossy networks (RPL) [12], the full network stack can easily be embedded in any constrained device. Therefore, it satisfies the IoT requirements where nodes are meant to be reliable, mostly battery-operated, and have constrained processing power. In TSCH, time is divided in small timeunits called timeslots. For each node that composes the network, the physical layer of the network stack is accessed through a slotframe structure as illustrated in Figure 1. A slotframe is a group of timeslots that repeats periodically. The number of timeslots in a slotframe is flexible and can be configured by higher layers of the protocol stack. The whole slotframe is scheduled in order to coordinate communication among the network nodes and decides when to transmit, receive, or sleep. In this way, all nodes only turn-on when necessary and sleep otherwise to save energy. Each timeslot is usually $10 \mathrm{~ms}$ or $15 \mathrm{~ms}$ long, allowing a node to transmit a frame and receive its acknowledgement. The TSCH network needs to be synchronized to achieve reliable communication. It allows channel hopping where each successive timeslot uses a different channel according to a predefined hopping pattern. This allows to achieve resilience against multi-path fading and external interference. There are 16 channels to be used in case of IEEE 802.15.4 radio which provides data rate of $250 \mathrm{kbps}$.

A slotframe can contain dedicated slots (contention-free) as well as shared slots (contention-based). Dedicated slots are those which are exclusive to a single sender-receiver node pair, whereas shared slots are common to multiple senderreceiver pairs and broadcast communication. As shown in Figure 1, a common practice is to locate shared slots at the beginning of the slotframe. For example nodes $n_{1}$ and $n_{3}$ share a slot at channel 0 and timeslot 0 to forward their traffic to $n_{2}$ and $n_{8}$ respectively. Although IEEE $802.15 .4 \mathrm{e}$ specifies the TSCH scheduling schemes, however specific implementation and maintenance are left to users. The conventional way of implementing a scheduling scheme, as done in WirelessHART or ISA100.11a, is the centralized scheduling. In this scheme, a central entity such as the personal area network (PAN) coordinator collects the network information and its topology, computes the schedules and routes and propagates this information towards the nodes.

\section{TSCH Network Advertising and Formation}

The presence of the network is advertised by the nodes through the transmission of enhanced beacons (EBs). An EB is a frame that contains information related to channel hopping, synchronization of timeslots, slotframe size, and the TSCH network in general. Nodes looking to join the network continuously listen for EBs from other nodes and associate with network upon successful reception. Capturing beacons is complex in TSCH networks due to channel hopping, because nodes are not aware of the hopping pattern prior to joining the network. Therefore, they keep switching channel every fixed duration of time until an EB is received. Synchronization of clocks is the fundamental requirement for TSCH to sustain a reliable communication. It requires clock synchronization accuracy in microseconds. Usually, the PAN coordinator is the source of the clock synchronization for all the nodes. In case the network does not have any PAN coordinator, the time-source is chosen as defined by the standard.

\section{RELATED WORK}

There are many works in literature that present the problem of inter-network interference and coexistence especially between IEEE 802.15.4 and IEEE 802.11 networks [13]. Other works focus on the interaction between co-located IEEE 802.15.4 networks that overlap in communication and interference ranges [5] [14] [15].

Nordin et al. [14] studied multiple co-located IEEE 802.15.4 wireless PANs and try to quantify the impact on network performance. The authors show that when multiple PANs co-exist independently and unsynchronized, they result in beacon collisions. They demonstrate that network experiences considerable beacon loss and emphasize on the need to coordinate beacon scheduling through adaptive schemes in inter-network operations.

Feeney et al. [5] performed a comprehensive simulation study on the inter-network interactions between two colocated IEEE 802.15.4 beacon-enabled PANs. The authors reveal complex behaviors that show large and slow oscillations in throughput: slow deterioration and recovery. Authors pointed out that the networks experience slow oscillations in packet reception rate in different periods and sometime complete packet loss.

An other work that studied the inter-network interference between TSCH networks is given in [15]. In this work, a vari- 
able number of synchronized and unsynchronized instances of the TSCH networks are investigated experimentally. The results indicate that when there are multiple TSCH networks with high traffic load, they face collisions due to inter-network interference causing network performance to decrease. While this study tries to show the traditional metrics such as packet delivery ratio, we take a different approach and take into account successful communication ratio as the desired metric.

\section{Approach And Experiment SET-UP}

The evaluations are performed through simulations for which we use the Cooja network simulator which is part of the Contiki-OS [16], [17]. We use RPL-specific [12] approach, which is commonly preferred for IoT networks.

In RPL, the network nodes associate with a coordinator called the border router (BR). In a typical scalable sensor network deployment, all nodes are not directly interacting with the application. Instead, traffic is directed toward a sink node, acting as a gateway to the outside world. To emulate upward traffic configuration appearing in sensor networks deployments, packets are generated periodically from all nodes toward the border router. For our experiments, the traffic generation period is set to 1 second. To analyze the effect of intra- and inter-network interference, the simulated transmission success ratio is set to $100 \%$, meaning that the outside environment has no impact on the transmission. In this configuration, a connection will be unsuccessful only when interference created by other nodes is blocking the communication process. To evaluate the impact of interference in (intra) and between (inter) networks, we analyze all packets exchanged between the nodes including acknowledgments and EBs. The IEEE802.15.4-TSCH protocol schedules the slotframe in order to achieve reliable communication. Active research is ongoing to find the best scheduling algorithm but no specific scheduling algorithm is defined by the standard. However, a default slotframe schedule namely minimal TSCH (TSCH_min) is available, which is a basic schedule having a slotframe with only one shared slot per node. For our experiments, we use TSCH_min as configured in [11], thus with 11 timeslots. With only one shared slot available to transmit data and EBs, TSCH_min is prone to creating interference. In this study, we analyze interference resulting from the network, and interference created by the co-location of other networks. For a network or a group of networks, noninterfering connections (or Successful Connections (SC)) can be seen as a time-dependent process $S C(t)$ with $S C^{\prime}(x)=$ $\frac{d S C(x)}{d t}$ as derivative. A connection is successful when the communication between two nodes of the same network is not impacted by any other transmission occurring at the same time. To estimate the amount of non-successful connections in a network, we compare the previous defined value to the total number of connections $T C(t)$ with $T C^{\prime}(x)=\frac{d T C(x)}{d t}$ as derivative. We define the Connection Success Ratio (CSR)

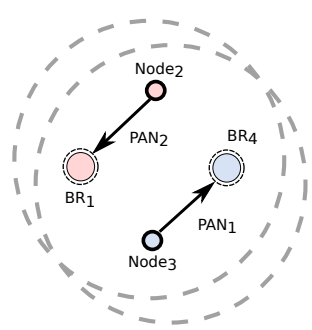

Figure 2. Two PANs topology, the dotted circles show the interference range and the communication range overlapping for both the networks.

of a network or several networks with:

$$
\operatorname{CSR}(x)=\frac{d S C(x)}{d T C(x)}
$$

A connection success ratio equals to 1 means, for the time $d t$ considered, all connections are successful. Similarly, a connection success ratio equals to 0 means, for the time $d t$ considered, all connections interfere. This ratio, representing the ratio of successful connections will be our metric to compare the amount of interference occurring in a network.

\section{Results AND Discussions}

The result section is divided into three sub-sections. In the first subsection, we use the CSR metric to analyze interference occurring in two simple co-located networks composed of two nodes each. In the second subsection, we increase the number of channels used to measure its impact in terms of interference mitigated. In the last subsection, we analyze the behavior of networks co-existing when the size of the network increases, and when the number of networks grows.

\section{A. Two PANs Topology}

Our first experimental scenario undertakes the impact of interference when two personal area networks (PANs) are closely co-located as depicted in Figure 2. The reason to use this stylized simple networks is to have minimal impact on link quality variations, the effects of varying interference, in-network contention, and unfairness for shared slots and demonstrate interference in different modes of interactions. We set both networks to be in each others' transmission range as well as interference range to see interesting behaviors. In the first part, only one channel is used in order to emphasize the interference situations. Both networks are synchronized based on the border-router's clock. Depending on the hardware used, these clocks can drift with time. Typical IEEE 802.15.4 devices having $32 \mathrm{MHz}$ clock with $30 \mathrm{ppm}$ may drift up to $60 \mu$ s per second [18]. In Figure 3, three different situations are considered.

- In Figure 3a, there is no drift between clocks. In this situation, there is a high probability of having no internetwork interference due to the random start-up offset of both border-routers. The connection success ratio of both networks seems to oscillate around 0.93 meaning that in average, $93 \%$ of all connections are successful.

- In Figure $3 b$, there is $1 \mu$ s per timeslots drift between clocks. In this situation, we observe periodic interference 


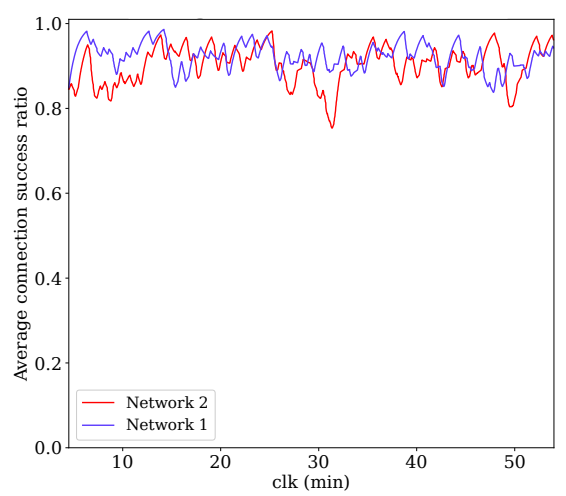

(a) No clock drift.

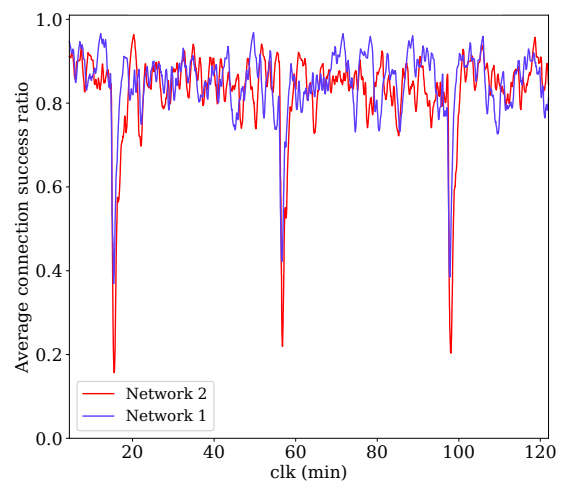

(b) $1 \mu \mathrm{s}$ per timeslot clock drift.

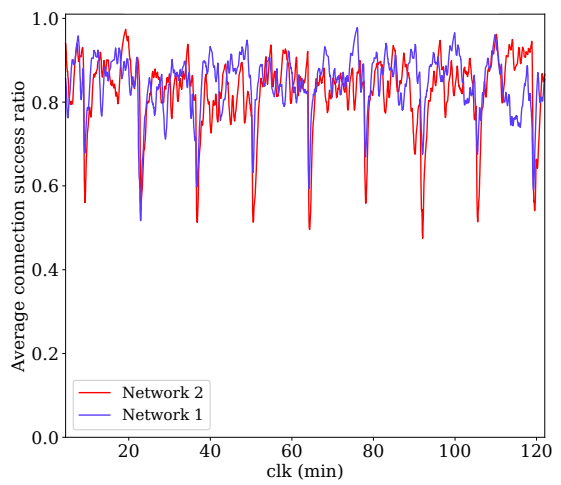

(c) $3 \mu$ s per timeslot clock drift.

Figure 3. Moving average of the connection success ratio of both TSCH networks with a random clock offset, and different clock drift situations.

every $\simeq 40 \mathrm{~min}$ represented by a drop of connection success ratio.

- In Figure $3 \mathrm{c}$, there is $3 \mu \mathrm{s}$ per timeslots drift between clocks and interference seems to occur more frequently.

When there is clock drift between the networks, interference seems to occur periodically. The interference observed is due to the co-location of both networks. When the clock drifts, both networks are artificially synchronized each periodic amount of time. Since TSCH_min provides only one opportunity to transmit per slotframe, this period can easily be calculated. It corresponds to the moment when the clocks are aligned on the first cell of the slotframe. With each timeslot measuring $T_{t s}=15000 \mu \mathrm{s}$ and the slotframe being $T_{s f}=N_{t s} \times T_{t s}$ long, with $N_{t s}$ the number of timeslots in the slotframe, the slotframes are synchronized every

$$
P=\frac{T_{t s}}{d} \times T_{s f}
$$

with $d$ the drift per timeslot. With $d=1$ and $N_{t s}=11$ for minimal TSCH, we obtain $P=41.25 \mathrm{~min}$ and with $d=3$, $P=13.75 \mathrm{~min}$. These values correspond to the observations of Figure $3 \mathrm{~b}$ and Figure $3 \mathrm{c}$.

Observations of Figure 3 show that inter-network interference is responsible for much more failed connections than intra-network interference. While intra-network interference seems to generate an average CSR of 0.93 in all experiments, inter-network interference generates a significant drop of average CSR. This drop is more significant when the clock drift is lower, because the connections are interfering during a longer time. However, when the clock drift is higher, the drops are occurring more often.

\section{B. Impact of Channel Hopping}

In this experiment, the same two PANs are used but the number of channels differs. Since inter-network interference occurs periodically, we analyze the CSR as a function of the timeslot difference between both networks. This timeslot difference is comprised between 0 (both networks clocks modulo $T_{t s}$ are equal) and 0.5 (both networks clocks modulo $T_{t s}$ differ by half a timeslot). The results are depicted in Figure 4 . We iterated this experiment three times and modified the number of channels used. The channel hopping pattern is random among the available channels.

With only 1 channel used, we observe that inter-network interference is occurring only when the timeslot difference is lower than $\simeq 0.12$ timeslot. We observe 0.3 to $0.7 \mathrm{CSR}$. After 0.15 timeslot difference, the CSR reaches its normal amount of 0.93 for this topology and schedule. This is due to the fact that the transmission time is approximatively 0.12 timeslot long. After this period, even if the timeslots are overlapping, the transmissions are not interfering. When increasing the number of channels used, the proportion of successful connections also increases. In the worst possible situation, that is when the timeslot difference is 0 with 4 channels, the average CSR raises to 0.55 . With 16 channels, the average CSR reaches 0.86 at 0 timeslot difference, which is close to the intra-network CSR measured of 0.93. This means that almost all the inter-network interference is avoided.

Increasing the number of channels allows to make full use of the channel hopping mechanism of TSCH. With channel hopping, intra-network interference still occurs at the same rate because of the structure of the minimal TSCH schedule. However, inter-network interference is avoided since two different networks may not be communicating on the same channel. In our case, the channel hopping pattern is random. Therefore, there is a theoretical probability $P(A)=\frac{1}{n_{c h}}$ that two transmissions occur on the same physical channel, $n_{c h}$ being the number of channels used. With more than 2 networks, this theoretical probability becomes $P\left(\bigcup_{i=1}^{n_{n e t w o r k s}-1} A_{i}\right)=$ $\sum_{k=1}^{n_{\text {networks }}-1}\left((-1)^{k-1} \sum_{I \in\left\{1, \ldots, n_{\text {networks }}-1\right\},|I|=k} P\left(A_{I}\right)\right)$ where $P\left(A_{i}\right)$ is the probability that a neighboring network interferes with the communication. Then, $P\left(A_{I}\right)=\frac{1}{\left(n_{c h}\right)^{|I|}}$. Therefore, having more available physical channels than co-located networks is crucial.

\section{Scalability}

In order to study the impact of scalability on inter-network interference, two different topologies are tested with only one channel in use. We first increase the number of nodes of each network. The drift is $1 \mu \mathrm{s}$ per timeslot. The results 


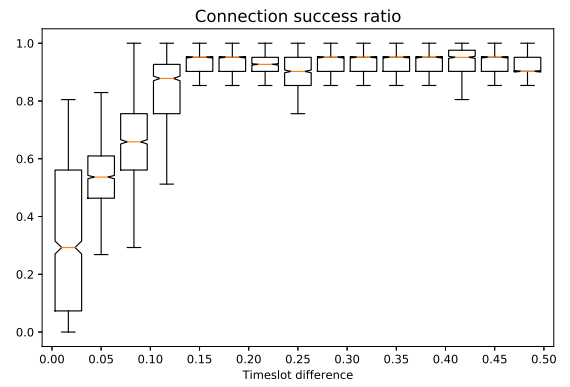

(a) 1 channel used.

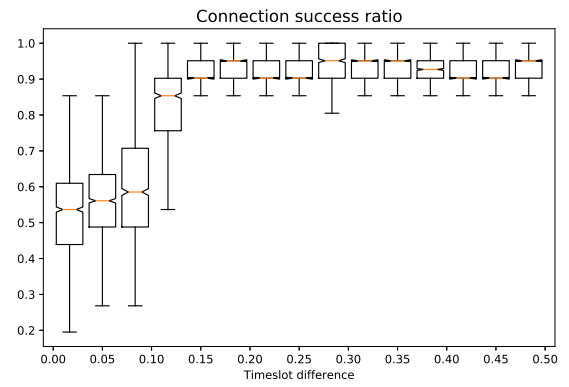

(b) 4 channels used.

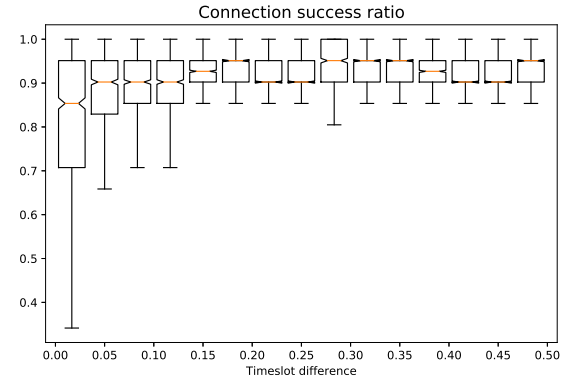

(c) 16 channels used.

Figure 4. Boxplot of the connection success ratio as a function of the timeslot difference between both networks.

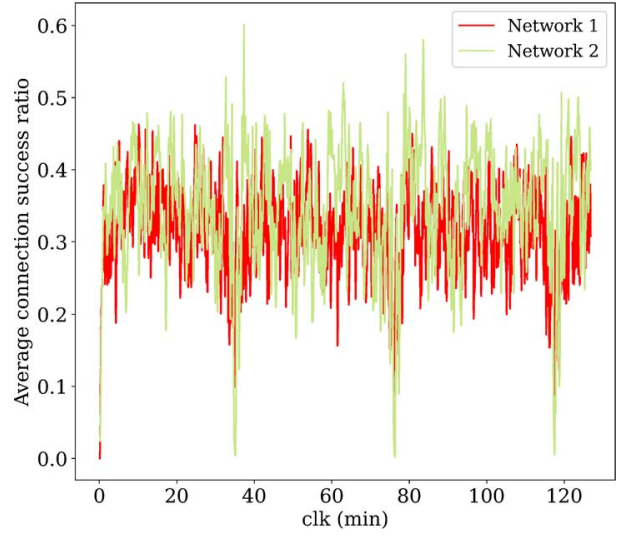

(a) Connection success ratio.

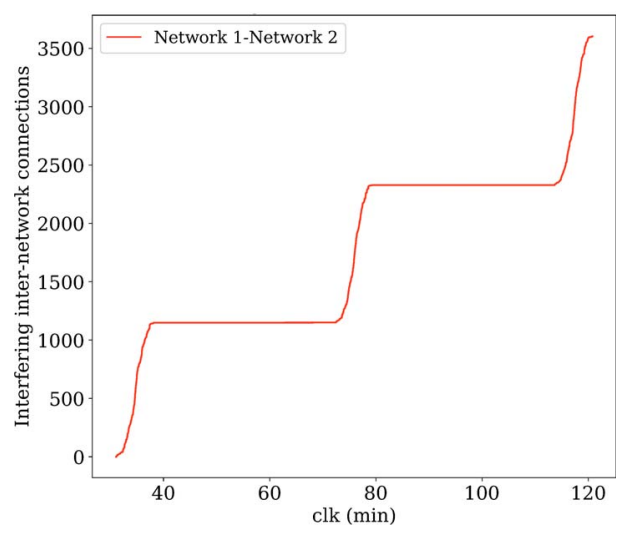

(b) Amount of interfering connections.

Figure 5. Experimental results of 2 networks of 4 nodes each.

are presented in Figure 5. With minimal TSCH schedule, increasing the number of nodes drastically increases the amount of interference within the network. Presented in Figure 5a, the average CSR oscillates around 32\%, meaning that only one packet out of 3 is successfully transmitted due to intra-network interference. We also observe a periodic drop of CSR due to inter-network interference. Similarly as with two nodes, both networks are artificially synchronized every $41 \mathrm{~min}$, creating this periodic pattern. This pattern is confirmed when observing the amount of interfering internetwork connections in Figure $5 \mathrm{~b}$.

While the pattern is similar to the first experiment, the amount of interfering connections is much more significant when the number of nodes increases. In this topology, when the networks are artificially synchronized, approx. 1000 connections interfere, while approx. 300 connections with only two nodes. In bigger networks, more messages are generated to successfully transmit data packets due to the design of the minimal TSCH slotframe. Coupled with routing messages generated by RPL, the amount of transmissions is more important in this topology.

We also analyzed four networks composed of two nodes in each network. In this configuration, every network is generated with a different drift, from 0 to $3 \mu$ s per timeslot for the Networks 1 to 4 respectively. This allows to emulate a real co-located TSCH deployment where different border- routers have different clocks. The amount of inter-network connections and CSR are presented in Figure 6. Due to the different drifts, each network interferes periodically with every other network. This behavior is represented in Figure 6a with periodic drop of CSR. However, since these networks are smaller, less packets are generated and interfering connections have less impact on the CSR metric. As depicted in Figure 6b, Network 1 and Network 2, which have a $1 \mu$ s clock drift between them, generate only 300 interfering connections, 3 times less than the previous experiment. We also notice that, the more frequent the inter-network interference occurs, the less interfering connections it creates. Indeed, clocks that align frequently are aligned for a shorter amount of time, reducing the probability to generate interfering connections.

From the two experiments with 4 nodes, it appears that increasing the number of networks generates much more inter-network interfering connections than only with two networks. After 2 hours of experiment with two networks, the total of inter-network interfering connection is $\simeq 3500$, while with 4 networks we reach 4400 .

\section{SUMmary}

In this paper, inter-network interference among several colocated TSCH networks is studied. With the CSR metric, we analyze the proportion of successful connections at each time. This metric shows that TSCH networks, scheduled with the minimal TSCH, experience significant interference within the 


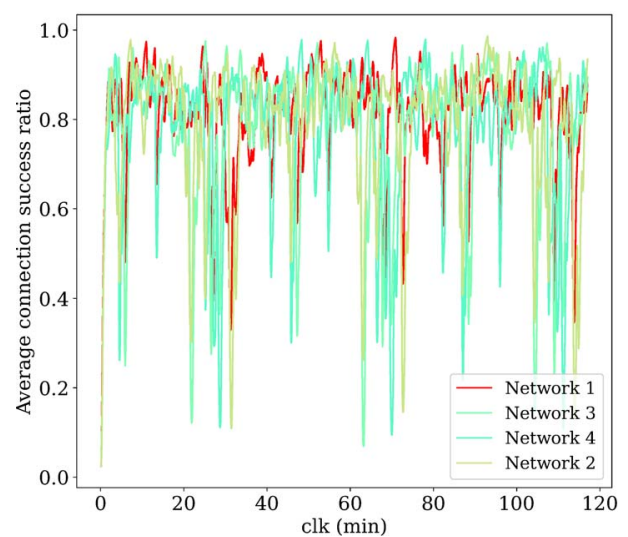

(a) Connection success ratio.

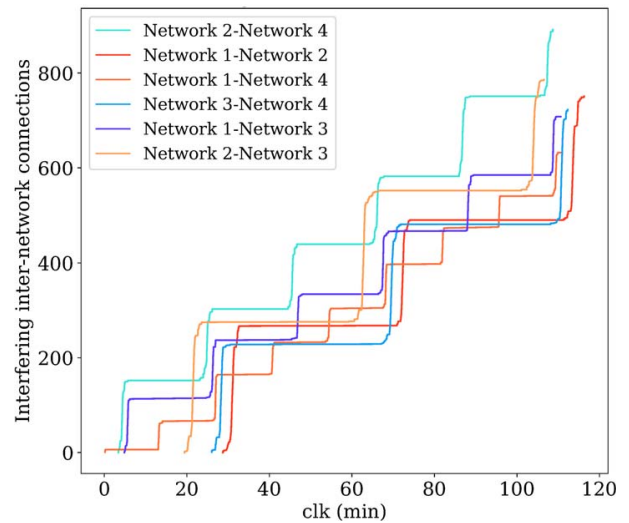

(b) Amount of interfering connections.

Figure 6. Experimental results of 4 networks of 2 nodes each.

network. Although, other scheduling algorithms may present better performance in terms of intra-network interference, yet the inter-network interference will still occur because both networks schedule communications on a slotframe which repeats over time. Co-located networks with the exact same clock will not interfere among each other if their timeslots are not aligned. However, in a real deployments, hardware clocks drift by a small amount and this creates periodic inter-network interference. When increasing the number of nodes, more interference appears periodically. Depending on the clock drift between both networks' coordinators, this may create significant down-time in the network. Increasing the number of networks causes inter-network interference to occur more frequently.

Co-locating TSCH networks becomes more challenging problem especially in industrial networks because different hardware vendors are unlikely to share their network stacks. To cope with this problem, our results show that all 16 channels must be used when configuring TSCH because this contributes greatly to reducing inter-network interference. Furthermore, the number of TSCH networks must be kept as small as possible. Ideally, all devices should be part of the same network and be synchronized on the same clock to avoid clock drift, however, this requirement is hard to fulfill in large scale deployments due to tight synchronization requirements of TSCH.

\section{ACKNOWLEDGMENT}

This research has been partially funded by the European Unions Horizon 2020 project INTER-IOT (grant number 687283).

\section{REFERENCES}

[1] IEEE Standard for Low-Rate Wireless Networks. IEEE Std 802.15.42015 (Revision of IEEE Std 802.15.4-2011), pages 1-709, April 2016.

[2] WirelessHART. https://fieldcommgroup.org. Last visited - 14.04.2018.

[3] The international society of automation- isa. https://www.isa.org/. Last visited - 28.04.2018.

[4] K. Kientopf, S. Raza, S. Lansing, and M. Güneş. Service management platform to support service migrations for iot smart city applications. In 2017 IEEE 28th Annual International Symposium on Personal, Indoor, and Mobile Radio Communications (PIMRC), pages 1-5, Oct 2017.
[5] L. M. Feeney, M. Frey, V. Fodor, and M. Gunes. Modes of internetwork interaction in beacon-enabled IEEE 802.15.4 networks. In 14th Annual Mediterranean Ad Hoc Networking Workshop (MED-HOCNET), pages 1-8, June 2015.

[6] I. Juc, O. Alphand, R. Guizzetti, M. Favre, and A. Duda. Energy consumption and performance of IEEE 802.15.4e TSCH and DSME. In IEEE Wireless Communications and Networking Conference, pages 1-7, April 2016.

[7] R. C. A. Alves and C. B. Margi. IEEE 802.15.4e TSCH mode performance analysis. In IEEE 13th International Conference on Mobile Ad Hoc and Sensor Systems (MASS), pages 361-362, Oct 2016.

[8] Ridha Soua and Pascale Minet. Multichannel assignment protocols in wireless sensor networks: A comprehensive survey. Pervasive and Mobile Computing, 16:2 - 21, 2015.

[9] IEEE Standard for Local and Metropolitan Area Networks-Part 15.4: Low-Rate Wireless Personal Area Networks (LR-WPANs). IEEE Std 802.15.4-2011 (Revision of IEEE Std 802.15.4-2006), pages 1-314, Sept 2011.

[10] T. Watteyne, M. Palattella, and L. Grieco. Using IEEE 802.15.4e timeslotted channel hopping (TSCH) in the internet of things (iot): Problem statement. Internet Requests for Comments, May 2015. http://www. rfc-editor.org/rfc/rfc7554.txt.

[11] X. Vilajosana, K. Pister, and T. Watteyne. Minimal 6tisch configuration draft-ietf-6tisch-minimal-19. Internet Requests for Comments, 25, Jan. 2017, work in progress.

[12] T. Winter, P. Thubert, A. Brandt, J. Hui, R. Kelsey, P. Levis, K. Pister, R. Struik, JP. Vasseur, and R. Alexander. Rpl: Ipv6 routing protocol for low-power and lossy networks. Internet Requests for Comments, March 2012. http://www.rfc-editor.org/rfc/rfc6550.txt.

[13] L. Angrisani, M. Bertocco, D. Fortin, and A. Sona. Experimental study of coexistence issues between IEEE $802.11 \mathrm{~b}$ and IEEE 802.15.4 wireless networks. IEEE Transactions on Instrumentation and Measurement, 57(8):1514-1523, Aug 2008.

[14] N. Nordin et al. Effects and implications of beacon collisions in colocated IEEE 802.15.4 networks. In 2012 IEEE Vehicular Technology Conference (VTC Fall), pages 1-5, Sept 2012.

[15] S. Ben Yaala, F. Theoleyre, and R. Bouallegue. Performance study of co-located IEEE 802.15.4-TSCH networks: Interference and coexistence. In 2016 IEEE Symposium on Computers and Communication (ISCC), pages 513-518, June 2016.

[16] A. Dunkels, B. Gronvall, and T. Voigt. Contiki - a lightweight and flexible operating system for tiny networked sensors. In 29th Annual IEEE International Conference on Local Computer Networks, pages 455-462, Nov 2004.

[17] Contiki: The open source os for the internet of things. http://www. contiki-os.org/. Last visited - 20.04.2018.

[18] D. Stanislowski, X. Vilajosana, Q. Wang, T. Watteyne, and K. S. J. Pister. Adaptive synchronization in IEEE 802.15.4e networks. IEEE Transactions on Industrial Informatics, 10(1):795-802, Feb 2014. 